\title{
共催化在有机合成反应中的研究进展
}

\author{
孙哲 何金梅* 屈孟男李㑆社* \\ (西安科技大学化学与化工学院 西安 710054)
}

\begin{abstract}
摘要 多种催化剂共催化的化学反应已成为提高合成效率的一种有效策略，日益受到国内外研究者的重视. 综述了近年来 有机合成中共催化反应的研究进展, 对金属-金属共催化、有机-有机共催化和金属-有机共催化三种主要类型进行了总结. 关键词 金属催化; 有机催化; 共催化; 有机合成
\end{abstract}

\section{Progress of Cooperative Catalysis in Organic Synthesis}

\author{
Sun, Zhe \\ He, Jinmei* \\ $\mathrm{Qu}$, Mengnan \\ Li, Kanshe* \\ (Department of Chemistry, Xi'an University of Science and Technology, Xi'an 710054)
}

\begin{abstract}
Cooperative catalysis has been widely considered as one of the most powerful strategy to improve the synthetic efficiency. The progress of cooperative catalytic in organic synthesis is reviewed, mainly including metal-metal, organic-organic and metal-organic cooperative catalysis.
\end{abstract}

Keywords metal catalysis; organo catalysis; cooperative catalysis; organic synthesis

有机合成中传统的单一催化体系通过特定催化剂 活化特定反应物, 从而降低反应能垒与另外一个未被活 化的反应物发生反应，达到提高反应效率的目的. 单一 催化体系对于特定反应具有显著作用，但也具有一定的 局限性. 因此, 设计和开发新的催化体系一直都是有机 合成领域的一大研究方向和热点. 其中, 两种或者两种 以上催化剂参与的共催化体系, 因其可实现原本不能或 者难以发生的反应, 具有高效、经济、绿色、操作简单 等优点而引起了化学家的极大兴趣, 在新药设计与合 成、天然产物合成和组合化学中都有广泛的应用 ${ }^{[1,2]}$.

共催化体系成功催化反应的关键是不同催化剂之 间能否和谐共存, 各司其职, 应避免催化剂之间形成强 的作用力而使其失去活性, 如硬酸-软碱、软酸-硬碱的 组合模式, 可有效地避免催化剂的失活. 根据催化剂与 反应物作用机理的不同, 共催化体系可分为四类, 如 Scheme 1 所示: (I)催化剂 A 和 B 分别活化不同的反应物 $\mathrm{R}_{1}$ 和 $\mathrm{R}_{2}$, 即协同催化, 协同催化也是共催化中最主要的 一类催化模式; (II)两个催化剂先后活化同一个反应物, $A$ 先将反应物 $R_{2}$ 活化为 $R_{2}{ }^{\prime}, B$ 再将 $R_{2}{ }^{\prime}$ 活化为 $R_{2}{ }^{\prime \prime}$, 该类
型也被称为串联催化; (III)反应物 $\mathrm{R}_{1}$ 与 $\mathrm{R}_{2}$ 形成中间体, 催化剂 $\mathrm{A}$ 和 $\mathrm{B}$ 同时作用于该中间体; (IV)两个催化剂同 时活化一个反应物, 共同将反应物 $\mathrm{R}_{2}$ 活化为 $\mathrm{R}_{2}{ }^{\prime}$. 根据 催化剂类型的不同，共催化又可分为金属-金属共催化、 有机-有机共催化和金属-有机共催化三种类型.

(I)

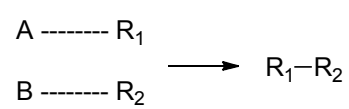

(II)<smiles>[R][R][R]([R7])[R7]</smiles>

(III)

(IV)
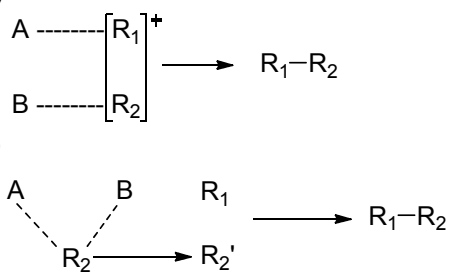

图式 1 共催化体系分类

Scheme 1 Classification of co-catalytic systems

\footnotetext{
*E-mail: jinmhe@xust.edu.cn

Received October 11, 2014; revised December 22, 2014; published online January 13, 2015.

Project supported by the National Natural Science Foundation of China (Nos. 21102113, 21473132, 51173145), the Natural Science Foundation of Schannxi Province (No. 2014JM2047) and the Shannxi Science and Technology Department (No. 2013KJXX-41).

国家自然科学基金(Nos. 21102113, 21473132, 51173145)、陕西省自然科学基金(No. 2014JM2047)及陕西省科学技术研究发展计划(No. 2013KJXX-41) 资助项目.
} 


\section{1 金属-金属共催化}

金属催化剂作为有机反应中的主要催化剂, 其研究 相对深入和成熟. 金属催化剂可高效地活化和构筑各类 $\mathrm{C}-\mathrm{H}$ 键、 $\mathrm{C}-\mathrm{C}$ 键、 $\mathrm{C}-\mathrm{O}$ 键及 $\mathrm{C}-\mathrm{N}$ 键等, 常被用于 催化加氢、脱氢、氧化、芳构化和异构化等反应, 在药 物分子、天然产物、液晶材料、染料和聚合物的合成中 均有广泛应用. 近年来, 两种或两种以上金属组成的金 属-金属共催化体系因可在一锅内活化多个化学键而高 效地构筑各类目标分子, 具有单一金属催化体系所不具 备的优点而受到化学家的青睐, 已成为催化领域中的又 一研究热点 ${ }^{[3]}$.

钯催化的烯丙基烷基化反应作为一类构筑各种化 学键的有效方法已被广泛地用于有机合成中, Feringa 研 究组 ${ }^{[4]}$ 利用共催化策略, 以金属 $\mathrm{Cu}$ 和 $\mathrm{Pd}$ 组成的共催化 体系, 通过 $\mathrm{Cu}$ 催化的甲基锌试剂对环已烯酮的不对称 1,4-加成以及串联的 Pd 催化的不对称烯丙基烷基化反 应, 成功地构筑了多取代的手性环己酮类化合物, 如 Scheme 2 所示.

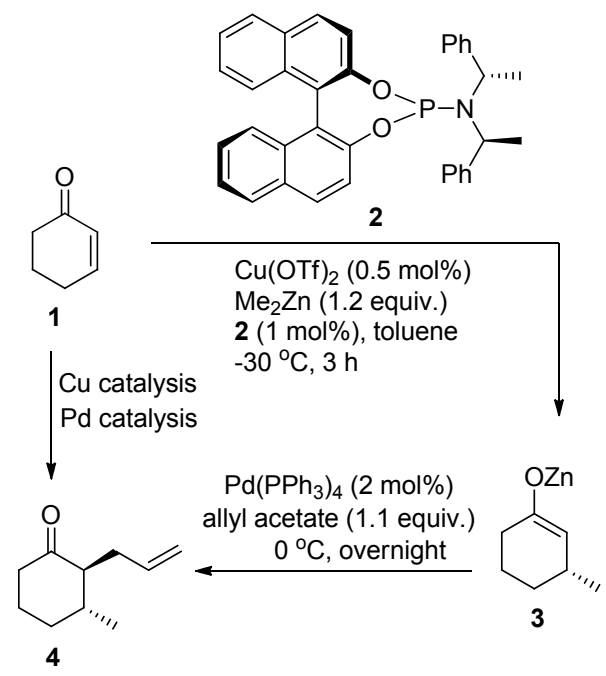

图式 2 铜和钯共催化的串联不对称共轭加成一烯丙基取代反 应

Scheme 2 Tandem asymmetric conjugate addition-allylic substitution reaction catalyzed by $\mathrm{Cu}$ and $\mathrm{Pd}$

利用过渡金属能够活化炔基的性质, 徐政虎研究 组 ${ }^{[5]}$ 以 $\mathrm{Au}$ 和 $\mathrm{Ga}$ 的共催化体系, 氨基炔烃和 $\alpha, \beta$-不饱和 酯为原料, 在温和条件下合成了具有重要生理活性的双 环[4.n.0]缩醛胺类化合物. 如 Scheme 3 所示, 在 $\mathrm{Au}$ 的催 化作用下, 反应物炔胺通过 exo-dig环化生成环烯胺, 随 后 $\mathrm{Ga}$ 作为 Lewis 酸与不饱和羰基化合物 6 作用, 使其 与环烯胺中间体发生串联的反电子需求的氧杂 DielsAlder 反应.

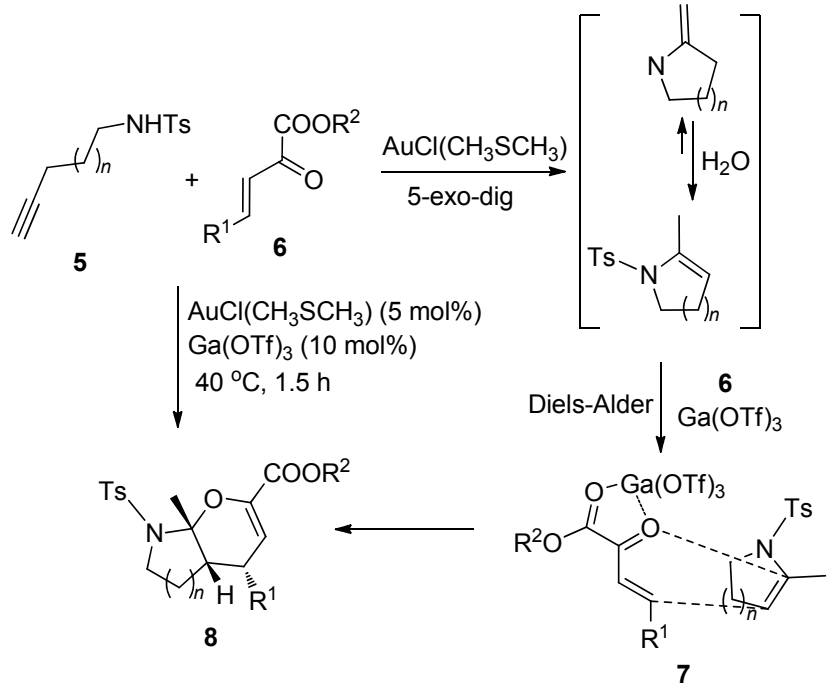

图式 3 金和镓共催化合成双环缩醛胺类化合物

Scheme 3 Synthesis of fused bicyclic aminals catalyzed by $\mathrm{Au}$ and $\mathrm{Ga}$

同样，利用过渡金属催化的炔的胺化反应，刘学伟 研究组 ${ }^{[6]}$ 报道了以 $\mathrm{Cu}(\mathrm{OAc})_{2}$ 和 $\mathrm{FePO}_{4}$ 的共催化体系, 通 过 2-氨基吡啶和炔烃的二胺化反应, 以较高的化学选择 性和区域选择性得到了具有重要生物活性的咪唑并 [1,2- $a$ 吡啶类化合物 11, 如 Eq. 1 所示. 该反应中, $\mathrm{Cu}(\mathrm{II})$ 活化炔键并与 2-氨基吡啶中的氮原子配位，通过分子间 胺化成环, $\mathrm{FePO}_{4}$ 作为氧化剂使 $\mathrm{Cu}(\mathrm{I})$ 复原为 $\mathrm{Cu}(\mathrm{II})$ 完成 催化循环.

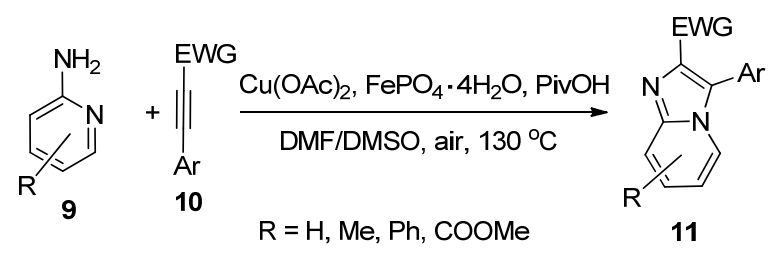

过渡金属可实现碳氢化合物与 $\mathrm{C}-\mathrm{C}, \mathrm{C}-\mathrm{N}$ 和 $\mathrm{C}-$ $\mathrm{O}$ 等多重键的直接碳氢键活化及加成反应，是构筑各类 化学键最经济、最高效的方法之一 ${ }^{[7]}$. Nakao 研究组 ${ }^{[8]}$ 曾报道了利用金属 $\mathrm{Ni}$ 和 $\mathrm{Zn}(\mathrm{Me})_{2}$ 或 $\mathrm{Al}(\mathrm{Me})_{3}$ 类金属 Lewis 酸组成的共催化体系, 实现了吡啶 2 位碳对炔烃 的加成反应, 可构筑 2-烯基取代的吡啶衍生物. 利用类 似的策略, Ong 研究组 ${ }^{[9]}$ 也报道了 $\mathrm{Ni}$ 和 $\mathrm{Al}$ 共催化的苯并 咪唑对苯乙烯的加成反应, 如 Scheme 4 所示. 研究表 明, 当向反应体系中加入 Lewis 酸 $\mathrm{AlMe}_{3}$ 时, 通过位阻 控制主要生成线性产物 15, 如无 $\mathrm{AlMe}_{3}$, 则由电子效应 控制生成支链产物 $\mathbf{1 6}$.

过渡金属催化的碳亲核试剂对氮杂苯并降冰片烯 的开环反应是合成氢化䒺衍生物的有效方法之一, 樊保 敏研究组 ${ }^{[10]}$ 近期报道了利用 $\mathrm{Cu}$ 和 $\mathrm{Pd}$ 的共催化体系, 以 


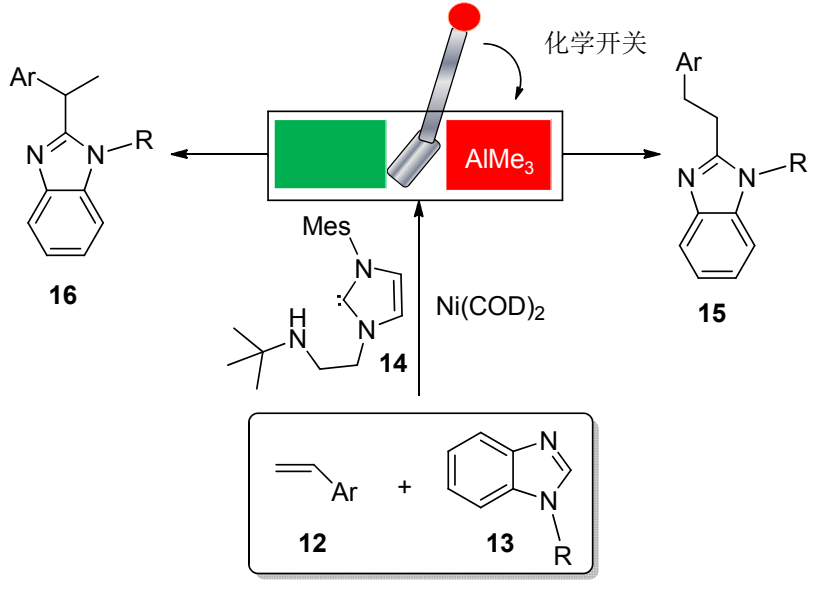

图式 4 镍和铝共催化的苯并咪唑与苯乙烯的区域选择性加 成反应

Scheme 4 Regioselective addition reaction of benzimidazole and styrene catalyzed by $\mathrm{Ni}$ and $\mathrm{Al}$
末端炔烃和氮杂苯并降冰片烯为原料，通过两类金属催 化剂协同催化的历程，实现了乙炔型碳亲核试剂对氮杂 苯并降冰片烯的加成开环反应，高效地得到了氢化䒺骨 架上含有炔基的开环产物 22, 如 Scheme 5 所示.

重氮化合物在过渡金属条件下分解形成金属卡宾 是获得卡宾的一个重要方法, 胡文浩研究组 ${ }^{[11]}$ 利用 $\mathrm{Rh}_{2}(\mathrm{OAc})_{4}$ 和 $\mathrm{AgOTf}$ 为共催化剂, 以 $\alpha$-重氮酯、2-炔基 芳醛亚胺及醇或水为原料, 通过串联的环化-三组分反 应，以较高的产率获得了 1,2-二氢异喹啉类化合物. 如 Scheme 6 所示, 反应物 $\alpha$-重氮酯与 $\mathrm{Rh}_{2}(\mathrm{OAc})_{4}$ 作用形成 金属卡宾 26, 醇 24 作为亲核试剂与金属卡宾反应生成 着基叶立德 27, 反应物 2-炔基芳醛亚胺在 AgOTf 的作 用下通过环化反应形成中间体 28 , 最终不稳定的叶立 德 27 被中间体 28 捕获得到 1,2-二氢异喹啉类化合物 30.

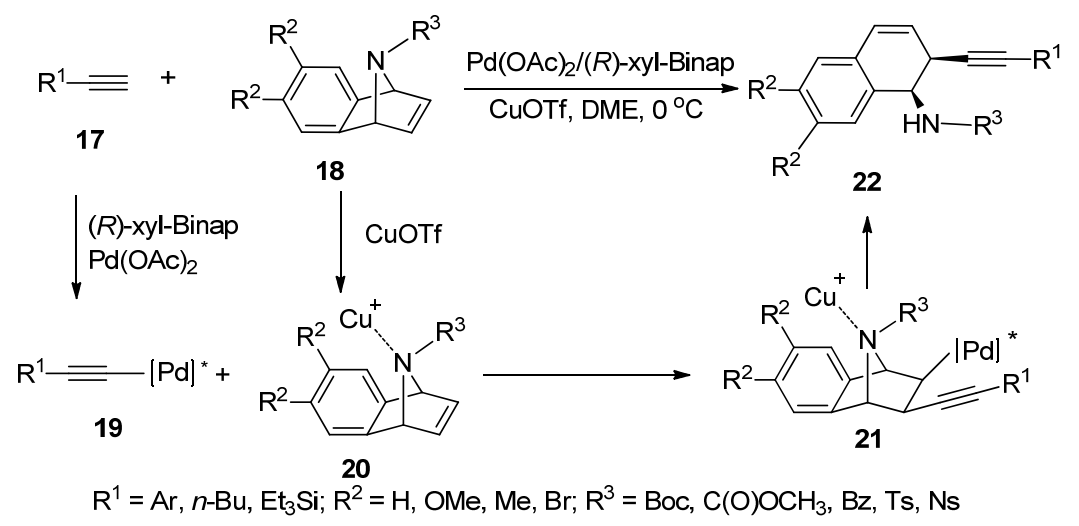

图式 5 铜和钯共催化的氮杂苯并降冰片烯与末端炔烃的不对称开环反应

Scheme 5 Asymmetric ring opening reaction of aza-benzonorbornadienes and terminal alkynes catalyzed by $\mathrm{Cu}$ and $\mathrm{Pd}$

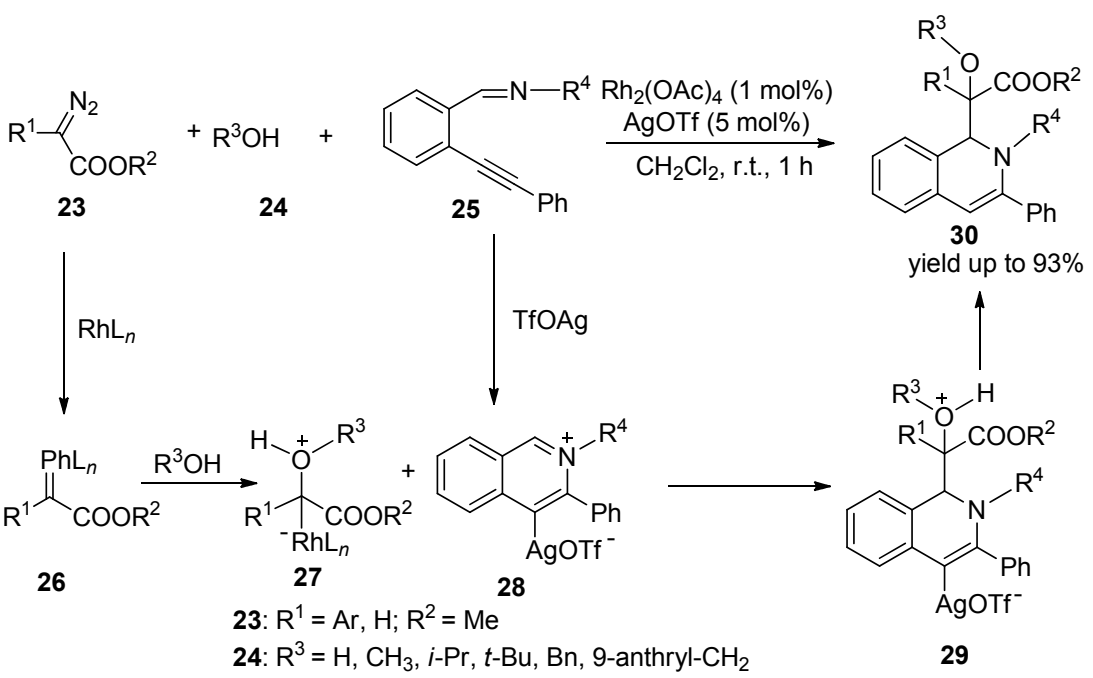

图式 6 醋酸铑和三氟甲烷磺酸银共催化的三组分串联环化反应

Scheme 6 Tandem three-component cyclization reaction catalyzed by $\mathrm{Rh}_{2}(\mathrm{OAc})_{4}$ and $\mathrm{AgOTf}$ 
金属一金属共催化具有高效和高对映选择性等优点, 可通过加成、偶联、缩合等反应构筑各类化学键. 其催 化体系的种类也非常丰富和多样, 如 $\mathrm{Cu}-\mathrm{Pd}$ 共催化、 $\mathrm{Cu}-\mathrm{Fe}$ 共催化 ${ }^{[12,13]} 、 \mathrm{Ir}-\mathrm{Ru}$ 共催化 ${ }^{[14]} 、 \mathrm{Au}-\mathrm{Ag}$ 共催化 ${ }^{[15]}$ 、 Co-Ti 共催化 ${ }^{[16]} 、 \mathrm{Ni}-\mathrm{Al}$ 共催化、 Ru-Pd 共催化 ${ }^{[17]}$ 等体系 在有机合成中都有很好的应用.

\section{2 有机一有机共催化}

自本世纪初至今, 有机小分子催化作为一个新兴的、 正在蓬勃发展的研究方向已取得了丰硕的成果 ${ }^{[18]}$, 常见 的有机催化剂有有机胺、有机膦、硫脲、氮杂环卡宾及 手性醇类质子型催化剂等; 按照催化机理又可分为烯胺 活化、亚胺离子活化、氢键活化、单电子占有轨道(SOMO) 活化、手性相转移催化剂活化和氮杂环卡宾活化等. 有机 催化因具有反应条件温和、催化剂容易制备和修饰、选 择性高等优点而受到研究者的青睐, 对有机催化的深入 研究也在不断开展. 将在一定条件下可兼容的不同类型 有机催化剂进行 “组合”, 通过有机一有机共催化模式来 提高合成效率已成为催化领域的又一研究方向.

\section{1 有机胺-硫腿共催化}

自 2000 年 List 等 $^{[19]}$ 报道了 $L$-脯氨酸催化的不对称 aldol 缩合反应, 有机胺作为一类重要的活化羰基的有 机催化剂通过烯胺活化可提高羰基化合物的亲核性能. 而手性硫脲作为有机催化剂的一员, 通过与反应物形成 氢键, 使活化部位的电子云密度或过渡态的 LUMO 能 量降低，从而有利于亲核试剂的进攻. 选择合适的有机 胺和硫嫝的共催化组合, 通过分别活化反应体系中的亲 核试剂和亲电试剂, 可成功地提高反应效率. 例如, Melchiorre 研究组 ${ }^{[20]}$ 于 2013 年报道了利用双官能团的 手性伯胺-硫脲催化剂, 通过协同催化的模式, 实现了 3-甲基环己烯酮与 $\alpha$-羰基酯的不对称插烯 aldol 缩合反 应, 如 Eq. 2 所示. 该双催化体系中, 催化剂 $\mathbf{3 3}$ 的氨基 与 3-甲基环已烯酮形成二烯胺使其活化, 而硫嫝位点则 通过氢键作用活化 $\alpha$-羰基酯.

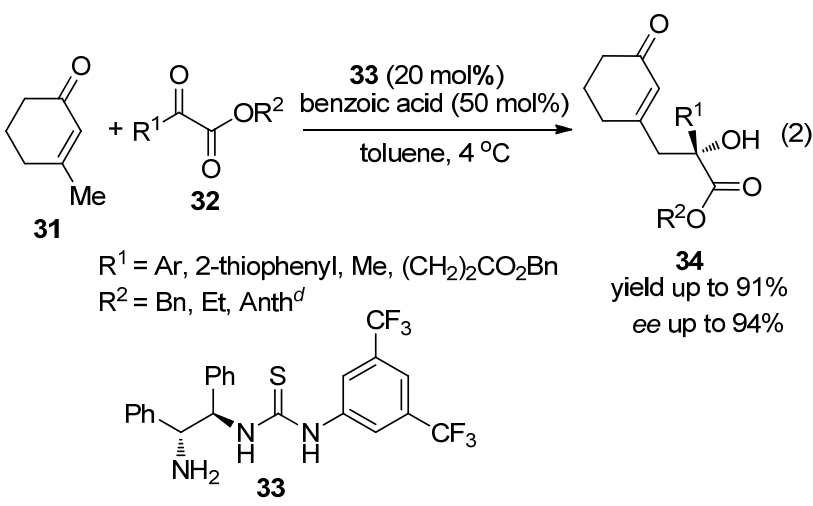

4-二甲氨基吡啶(DMAP)因能有效地催化高位阻、 低反应性的醇和胺的酰化、羰基化、醚化及酯化等反应， 已成为有机合成中最常用的催化剂之一. 鉴于 DMAP 优异的催化性能, 2011 年, Seidel 研究组 ${ }^{[21]}$ 报道了利用 DMAP 和手性酰胺硫脲为共催化剂, 以苯甲酸酐为酰化 试剂, 实现了内消旋邻二胺的选择性酰胺化, 如 Eq. 3 所示. 该共催化体系中, DMAP 活化酸酐生成非手性的 $N$-酰基吡啶阳离子，该阳离子与手性硫脲形成离子对， 最终以较高的对映选择性实现了内消旋邻二胺的选择 性酰化, 该研究为合成各类手性邻二胺型配体和药物中 间体提供了有效方法.

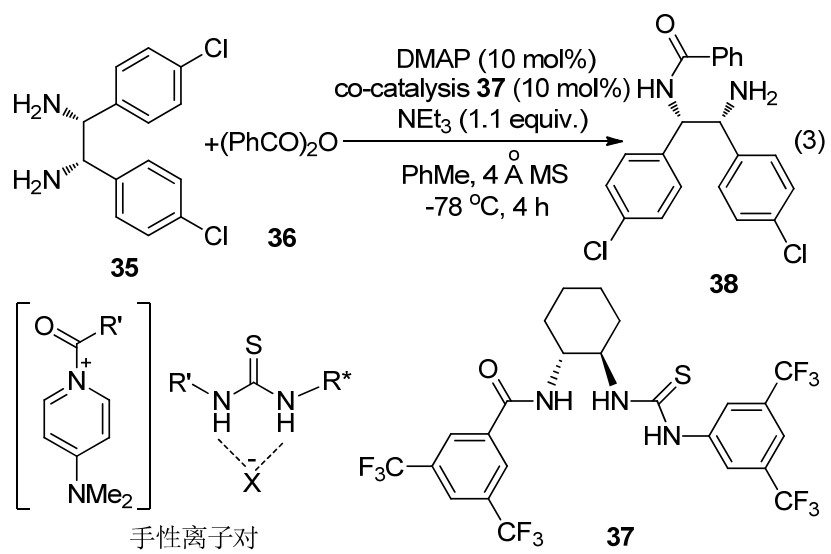

\section{2 有机胺-有机磷酸共催化}

有机磷酸作为一类具有较强酸性的有机小分子催 化剂，磷原子上所连的羟基可作为 Brønsted 酸提供质子 或与底物形成氢键，磷原子上的双键氧又可作为碱性位 点提供孤对电子，因此有机磷酸类化合物本身具有双活 化的特点. 又由于手性磷酸分子中磷原子具有一定的立 体构型，通过调节磷原子周围的取代基可改变磷酸在催 化过程中的立体选择性，从而实现高对映选择性合成.

Toste 研究组 ${ }^{[22]}$ 曾报道了利用手性磷酸 41 和氨基酸 酯 42 共催化的不对称氟化反应. 如 Eq. 4 所示, 氨基酸 酯活化羰基使其生成烯胺，手性磷酸作为相转移催化剂 与亲电性氟化试剂 Selectfluor 形成离子对, 成功实现了 羰基 $\alpha$ 位的不对称氟化.
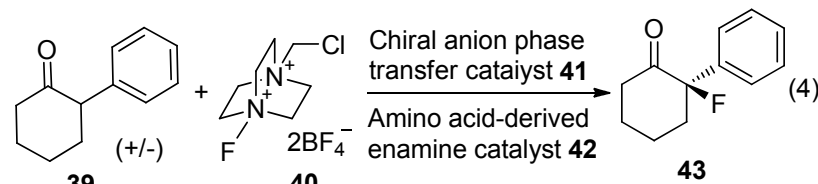

39

40

43
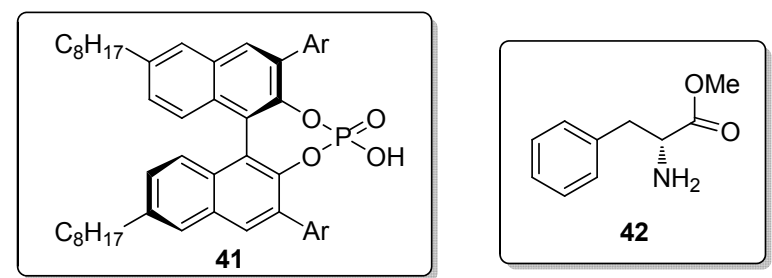


\section{3 有机胺一氮杂环卡宾共催化}

氮杂环卡宾作为一类重要的有机催化剂, 可将醛类 化合物的反应极性发生翻转, 使其由亲电性转化为亲核 性, 如氮杂环卡宾催化的安息香缩合反应 ${ }^{[23,24]}$ 及 Stetter 反应 ${ }^{[25 \sim 27]} .2009$ 年, Rovis 研究组 ${ }^{[28]}$ 以手性二级胺 46 和 三唑类氮杂环卡宾 48 为共催化剂, 通过二级胺催化的 不对称迈克尔加成反应和串联的分子内安息香缩合反 应，高效地构筑了多取代且手性碳原子密集的 2-羟基环 戊酮类化合物，如 Scheme 7 所示.

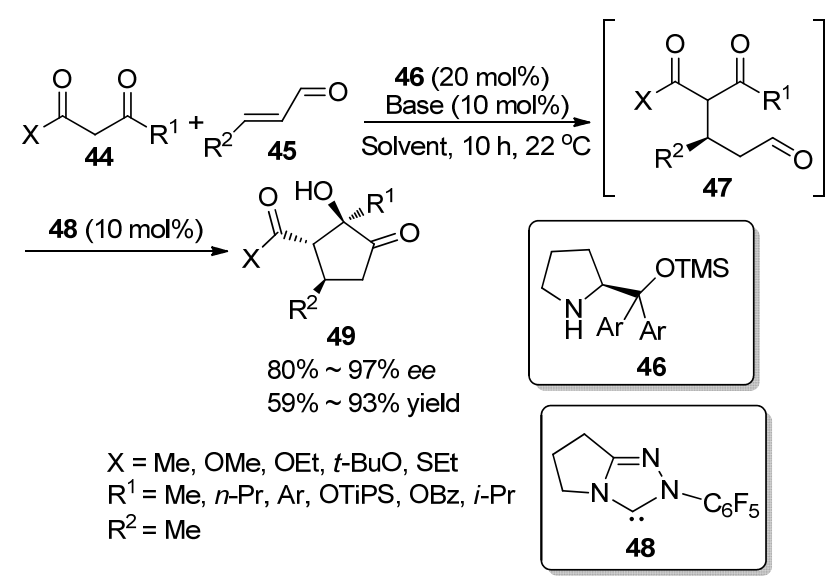

图式 7 手性二级胺和氮杂环卡宾共催化合成官能团化环戊 酮化合物

Scheme 7 Synthesis of functionalized cyclopentanones catalyzed by secondary amine and NHCs

\section{4 氮杂环卡宾-有机羧酸共催化}

Rovis 研究组致力于氮杂环卡宾作为有机催化剂的 多样性研究, 随后该研究组又报道了利用氮杂环卡宾和 Brønsted 酸共催化构筑 $\gamma$-内酰胺的反应. 如 Scheme 8 所 示, 三唑类氮杂环卡宾催化剂 $\mathbf{5 2}$ 将反应物 $\alpha, \beta$-不饱和醛 50 的反应极性翻转, 使其由 $\mathrm{a}^{3}$ 合成子转化为亲核性的 $\mathrm{d}^{3}$ 合成子 ${ }^{[29,30]}$. 同时, 邻氯苯甲酸通过氢键作用活化不 饱和亚胺 51, 两个被活化的反应物通过类 aldol 缩合反 应及串联的环化反应得到 $\gamma$-内酰胺 $\mathbf{5 4}^{[31]}$. 考虑到两类 催化剂的酸碱兼容性, 反应中选择邻氯苯甲酸的钠盐为 羧酸催化剂的前体, 邻氯苯甲酸钠与三唑盐作用产生氮 杂环卡宾, 自身得到质子成为 Brønsted 酸活化不饱和 亚胺.

如上文所述, 有机一有机共催化体系主要通过协同 催化模式, 在温和条件下高选择性地催化各类反应. 有 机小分子催化剂之间的有效 “组合”, 如有机胺-硫䐂共 催化 ${ }^{[32]}$ 、有机胺一磷酸共催化、有机胺一氮杂环卡宾共催 化 ${ }^{[33]}$ 、有机胺-相转移催化剂共催化等体系中, 各种催 化剂既保持了原有的催化特性, 相互之间又具有较好的 兼容性, 具有催化机理明确等特点. 因此, 相信在将来
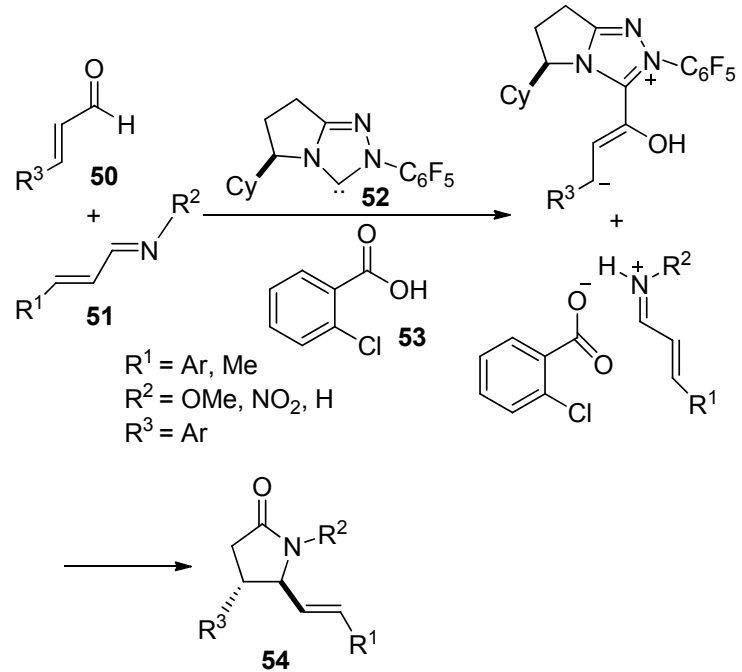

up to $99 \%$ yield $93 \%$ ee@ $f 20 / 1 \mathrm{dr}$

图式 8 氮杂环卡宾和 Brønsted 酸共催化合成 $\gamma$-内酰胺 Scheme 8 Synthesis of trans- $\gamma$-lactams catalyzed by NHCs and Brønsted acid

会有更多类型的有机一有机共催化体系被开发和应用.

\section{3 金属-有机共催化}

金属催化剂和有机小分子催化剂作为有机合成中 最主要的两类催化剂, 都已得到较为深入的研究, 而将 金属催化剂和有机小分子催化剂 “组合” 的共催化体系, 取二者之长, 具有有机催化的高选择性和金属催化的高 效性双重优势，可使反应更加多样性和高效，为有机合 成提供了更多的高反应活性和高选择性的方法 ${ }^{[34 \sim 36]}$. 金属-有机共催化体系的关键为两类催化剂在同一体系 中的兼容性问题，选择合适的有机小分子催化剂，使其 不与金属催化剂形成强的配位键而失去活性, 才可有效 地催化反应, 因此金属-有机共催化体系的研究充满了 挑战.

\section{1 金属-有机胺共催化}

众所周知，有机胺作为有机小分子催化中的一类重 要催化剂, 通过与羰基作用活化反应物. 利用有机胺和 金属共催化的策略, Ibrahem 研究组 ${ }^{[37,38]}$ 报道了以 $\mathrm{Pd}$ 和 脯氨酸衍生物组成的共催化体系, $\alpha, \beta$-不饱和醛和芳基 硼酸为原料，通过分子间的 1,4-加成反应，以较高的收 率和对映选择性得到了 $\beta$ 芳基醛 ${ }^{[39]}$ ，如 Scheme 9 所示. 随后, 该研究组利用类似的策略, 以 $\alpha, \beta$-不饱和醛和含 有活性亚甲基的烯丙基醋酸酯为原料，通过一锅内有机 胺催化的不对称迈克尔加成反应和串联的 Pd 催化的不 对称烯丙基烷基化反应，高效和高对映选择性地构筑了 多取代、手性碳原子密集的五元和六元环化合物 ${ }^{[0]}$, 如 
Eq. 5 所示.

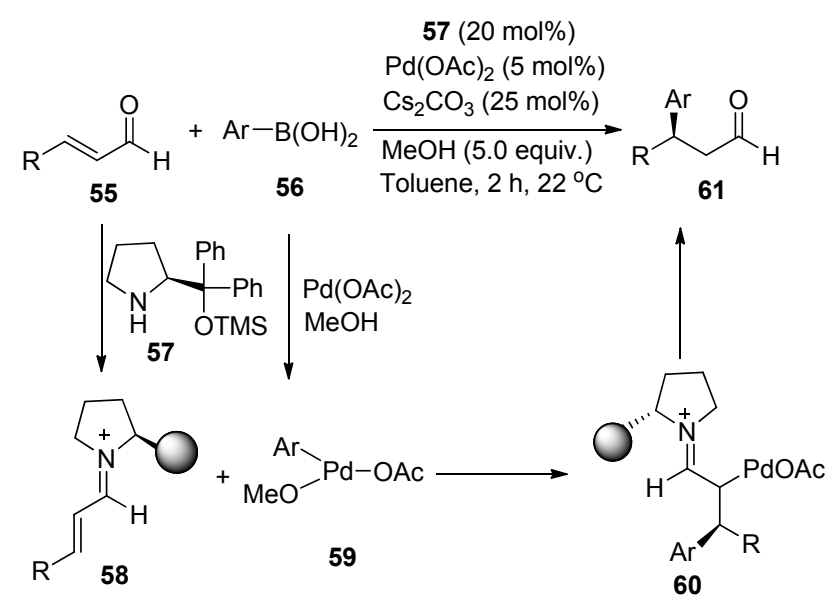

图式 9 手性胺和钯共催化的 $\alpha, \beta$-不饱和醛与芳基嗍酸的 $\beta$ 芳基化反应

Scheme $9 \beta$-Arylation of $\alpha, \beta$-unsaturated aldehydes and aryl boronic acids catalyzed by chiral amine and Pd

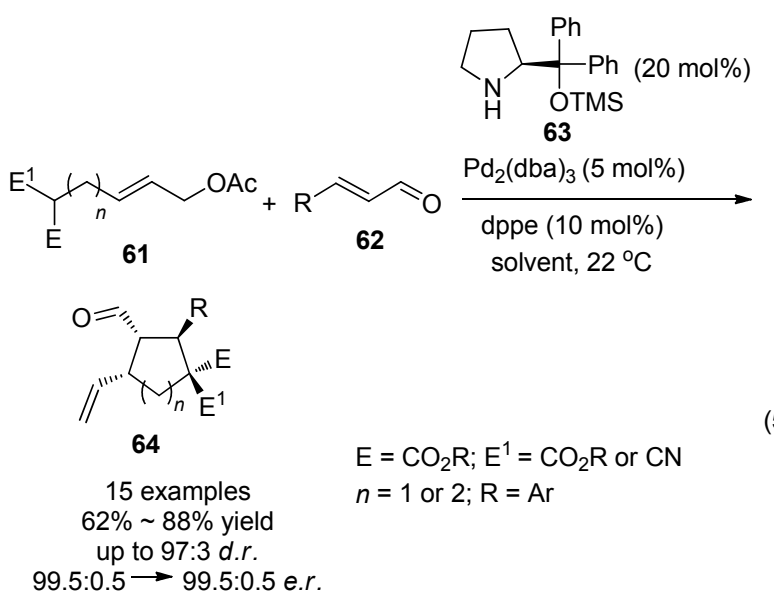

利用一锅内串联的 Heck-Aldol-Heck 多组分反应, 黄志真研究组 ${ }^{[41]}$ 以 $\mathrm{Pd}(\mathrm{OAc})_{2}$ 和吡咯的金属-有机共催化 体系, 以碘代芳烃、烯丙醇和多聚甲醛为原料, 有效地 构筑了三个新 $\mathrm{C}-\mathrm{C}$ 键, 获得了各类芳基取代的 $\alpha, \beta$-不 饱和醛, 如 Scheme 10 所示.

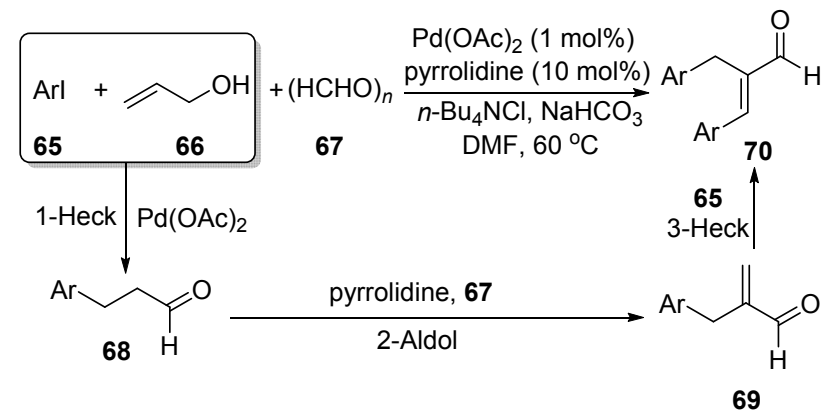

图式 10 钯和吡啶共催化的串联 Heck-Aldol-Heck 多组分反应 Scheme 10 Cascade Heck-Aldol-Heck reaction catalyzed by Pd and pyrrolidine
金属催化的加氢酰化反应是制备醛酮的有效方法, Douglas 研究组 ${ }^{[42]}$ 报道了利用金属 $\mathrm{Rh}$ 和 2-氨基吡啶 72 共催化的二取代烯烃的分子内加氢酰化反应，如 Scheme 11 所示. 2-氨基吡啶 72 作为有机催化剂与反应 物中的醛基作用生成亚胺中间体 73, 随后在金属 $\mathrm{Rh}$ 的 催化下通过分子内加氢酰化反应生成环状的酮类化合 物 74. 与金属单独催化的烯烃加氢酰化反应相比 ${ }^{[43 \sim 46]}$, 该金属-有机共催化体系无需在反应物中嵌入其他螯合 基团，并且可有效地避免醛的脱羰分解和烯烃的异构 化.

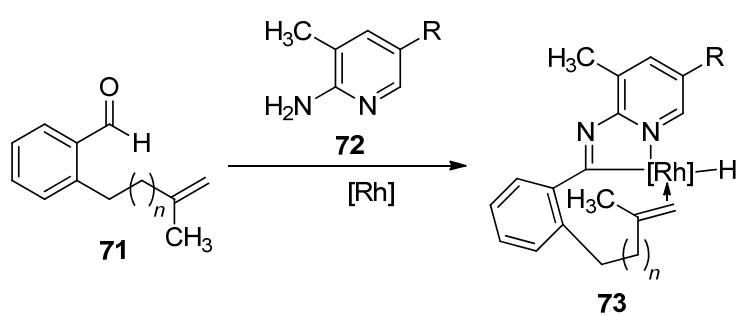

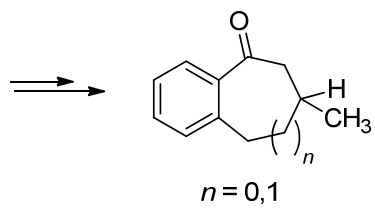

74

图式 11 铑和 2-氨基吡啶共催化的分子内加氢酰化反应 Scheme 11 Intramolecular hydroacylation catalyzed by Rh and 2-aminopyridine

金鸡钠碱作为有机催化剂被广泛地应用于不对称 合成中, Carreira 研究组 ${ }^{[47]}$ 于 2013 年报道了金鸡纳伯胺 和金属 $\mathrm{Ir}$ 共催化的支链醛的 $\alpha$-不对称烯丙基烷基化反 应, 如 Scheme 12 所示. 研究表明选择不同构型的金鸡 纳伯胺和金属配体, 可以高收率、高 $e e$ 值和较高的 $d r$ 值获得各种构型的产物.

\section{2 金属-磷酸共催化}

磷酸作为有机催化剂可通过氢键作用活化反应物, 提高反应物的亲电性. 胡文浩研究组 ${ }^{[48]}$ 利用共催化策 略, 以 $\mathrm{Rh}_{2}(\mathrm{OAc})_{4}$ 和 $\mathrm{BINOL}$ 衍生的手性磷酸为共催化 剂， $\alpha$-烷基- $N$-甲基重氮乙酰苯胺和亚胺为原料，以大于 99：1 的非对映选择性和高达 98\%的对映选择性成功地 获得了一类具有多个手性中心以及良好生理活性的 3取代吲哚酮衍生物. 如 Scheme13 所示, $\alpha$-烷基- $N$-甲基 重氮乙酰苯胺在 $\mathrm{Rh}_{2}(\mathrm{OAc})_{4}$ 的催化下分解形成金属卡 宾, 通过分子内 $\mathrm{C}-\mathrm{H}$ 键插入形成活性离子对中间体 $\mathbf{8 5}$, 最终该活性离子对被磷酸活化的亚胺捕捉. 该反应中磷 酸作为有机共催化剂, 既活化了亚胺, 又营造了手性环 境, 实现了不对称催化. 

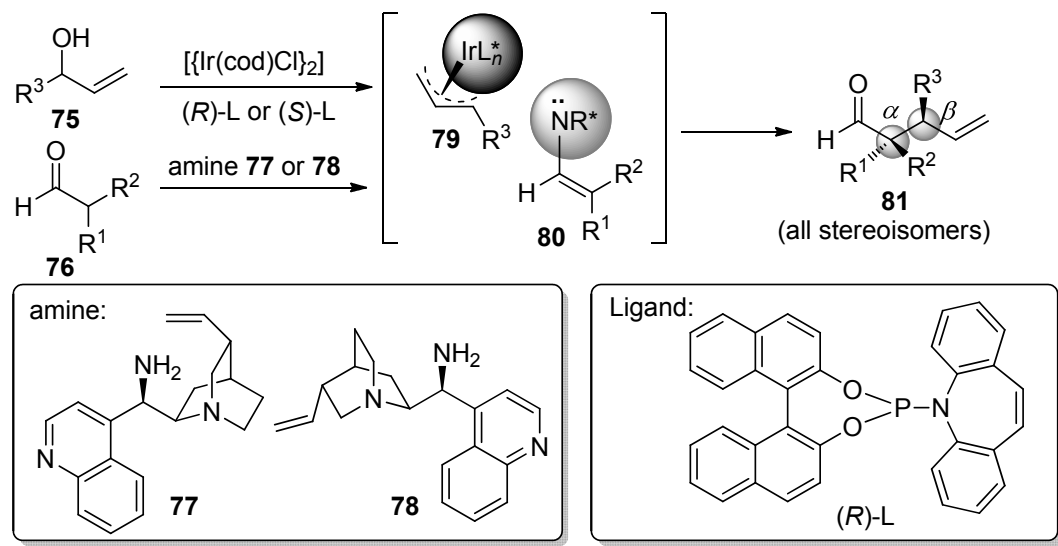

$\mathrm{R}^{1}=\mathrm{Me}, \mathrm{NBoc} ; \mathrm{R}^{2}=\mathrm{Pr}, \mathrm{Me}, \mathrm{CH}_{2} \mathrm{NO}_{2}, \mathrm{CH}_{2} \mathrm{OBz}, \mathrm{CH}_{2} \mathrm{Phth} ; \mathrm{R}^{3}=\mathrm{Ar}$

图式 12 铱和手性胺共催化支链醛的不对称 $\alpha$-烯丙基烷基化反应

Scheme $12 \alpha$-Allylation of branched aldehydes catalyzed by Ir and chiral amine

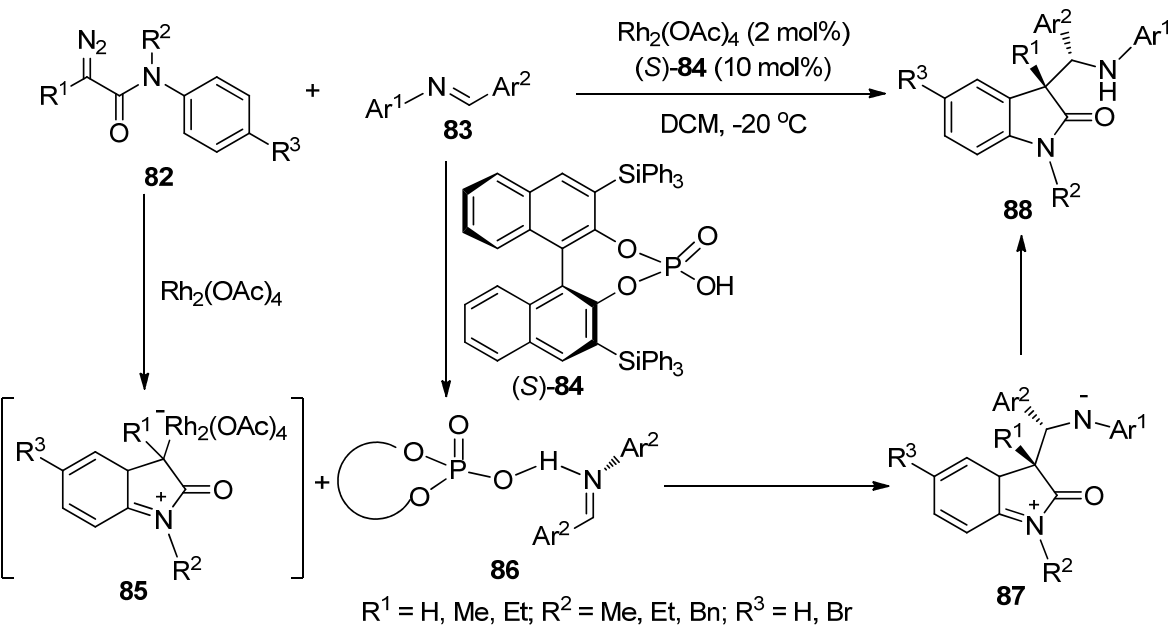

图式 13 醋酸铑和手性磷酸共催化 $\alpha$-烷基- $N$-烷基重氮乙酰苯胺和亚胺的不对称反应

Scheme 13 Asymmetric reaction of $\alpha$-alkyl- $N$-methyl-diazoacetamide and imine catalyzed by $\mathrm{Rh}_{2}(\mathrm{OAc})_{4}$ and chiral phosphoric acid

利用过渡金属 $\mathrm{Rh}$ 或 $\mathrm{Ru}$ 与有机磷酸的共催化体系, 胡文浩研究组还报道了一系列金属卡宾和亚胺参与，以 多组分 Mannich 反应为主要反应, 成功构筑手性环缩 醛 ${ }^{[49]} 、 a, \beta$-二氨酸衍生物 ${ }^{[50]}$ 及全取代四氢异喹琳衍生物 的方法 ${ }^{[51]}$. 例如, 以芳基重氮醋酸酯、芳胺、芐氧羰基 胺及 2-醛基肉桂酸酯为原料, 通过分子内 Mannich 反应 及串联的氮杂 Michael 加成反应, 以中等收率、高立体 选择性地构建了一系列骨架多样、结构丰富的含季碳的 全取代四氢异喹琳衍生物 ${ }^{[51]}$, 如 Scheme 14 所示. 其中, 芳基重氮醋酸酯与金属 $\mathrm{Ru}$ 生成金属卡宾, 而磷酸则通 过氢键作用活化中间体亚胺.

利用金属 $\mathrm{Ru}$ 和手性磷酸组成的共催化体系, 游书 力研究组 ${ }^{[52]}$ 曾报道了通过一锅内串联的烯烃复分解反 应和分子内不对称 Friedel-Crafts 烷基化反应, 高效和高 对映选择性构筑具有重要价值的多环吲哚类化合物的
反应, 如 Scheme 15 所示. 其中, 磷酸通过与中间体 $\mathbf{1 0 2}$ 中羰基的氢键作用增强了羰基 $\beta$ 碳原子的亲电性, 使其 更易与取代吲哚发生 Friedel-Crafts 反应.

\section{3 金属-硫脲共催化}

硫脲与有机磷酸相似, 可通过氢键作用活化反应 物. 利用金属催化剂和硫脲的共催化体系，以协同催化 的模式可有效地提高反应的效率和不对称选择性. Oh 研究组 ${ }^{[53]}$ 利用手性金属钴配合物和非手性硫脲为共催 化剂, 通过异氧基乙酸甲酯和醛的不对称羟醛缩合反 应, 以较高的 $e e$ 值和 $d r$ 值获得了多取代的噁唑啉类化 合物 108, 如 Eq. 6 所示. 其中, 硫腿通过氢键作用活化 异氧基乙酸甲酯，而手性金属钴配合物则作为 Lewis 酸 活化醛羰基, 使其以较高的立体选择性发生不对称羟醛 缩合反应. 


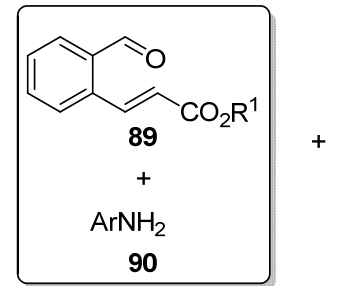

90<smiles>O=P(O)(O)Oc1c(Br)cc2ccccc2c1-c1c(Br)cc2ccccc2c1Br</smiles>

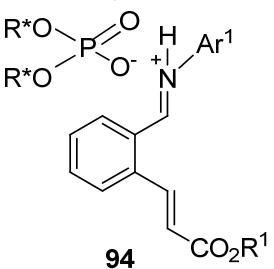

$$
\begin{gathered}
\mathrm{Ar}^{2} \stackrel{\mathrm{N}_{2}}{\mathrm{CO}_{2} \mathrm{CH}_{3}} \\
92 \\
+ \\
\mathrm{CbzNH}_{2} \\
91 \\
\hline
\end{gathered}
$$

$\left[\mathrm{RuCl}_{2}(p-\mathrm{cymmene})\right]_{2}(1 \mathrm{~mol} \%)$ $(R)-93(5 \mathrm{~mol} \%)$ $t$-BuOK

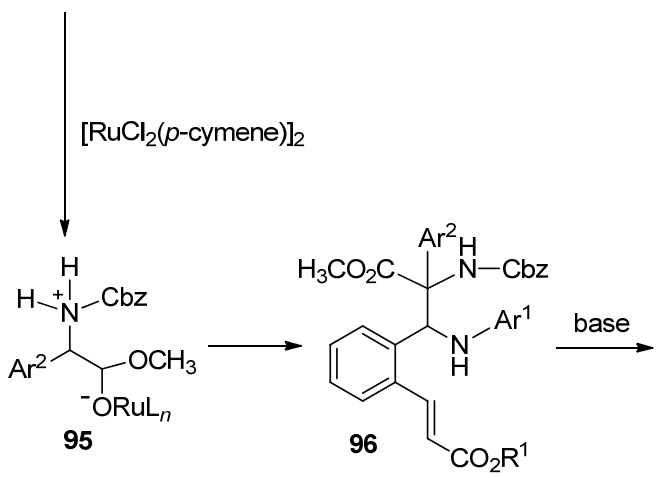<smiles>CC(=O)N1C(N[Al])c2ccccc2[C@H]1C(=O)O</smiles><smiles>C1CC1</smiles>

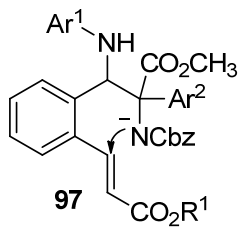

图式 14 钉(II)和手性磷酸共催化的对映选择性四组分串联氮杂迈克尔加成反应

Scheme 14 Enantioselective four-component cascade aza-Michael addition reaction catalyzed by Ru(II) and chiral phosphoric acid

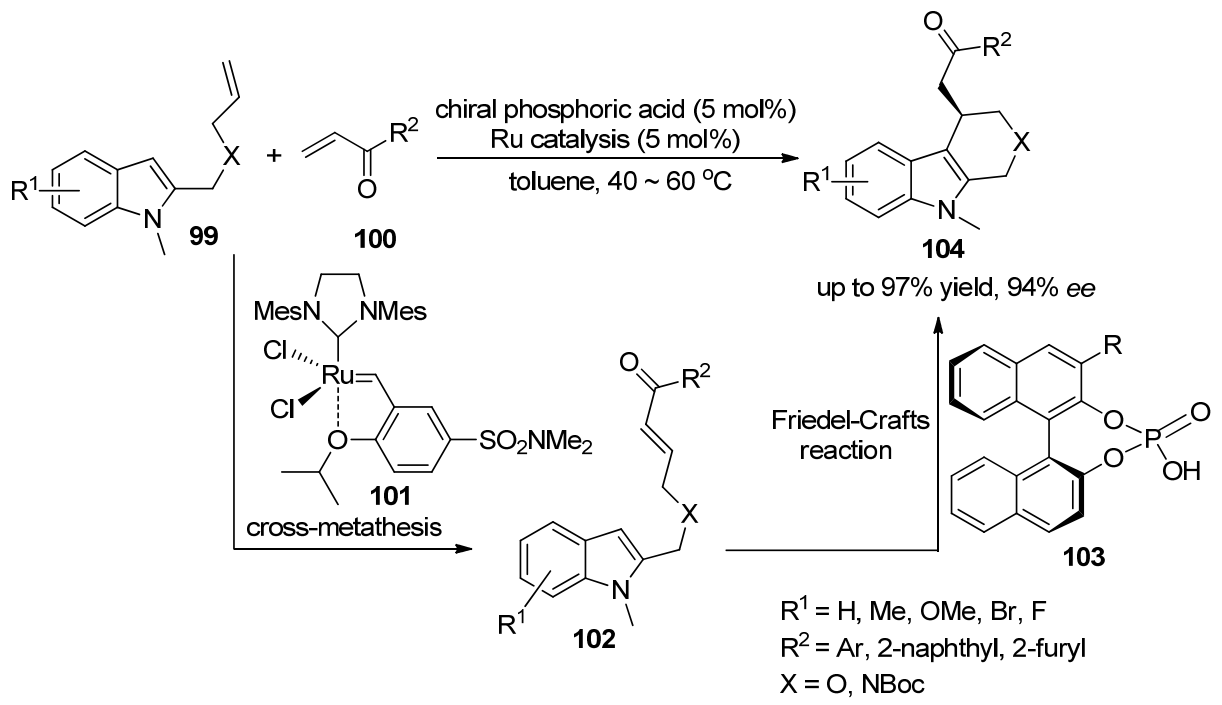

图式 15 钉和手性磷酸共催化的烯烃复分解反应和不对称 Friedel-Crafts 烷基化串联反应

Scheme 15 Olefin cross-metathesis and asymmetric Friedel-Crafts alkylation reaction catalyzed by Ru and chiral phosphoric acid

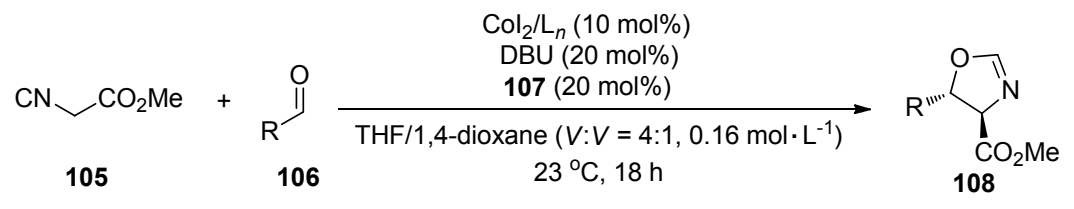<smiles>CN(C(=S)N([Tl])c1cc(C(F)(F)F)cc(C(F)(F)F)c1)c1cc(C(F)(F)F)cc(C(F)(F)F)c1</smiles>

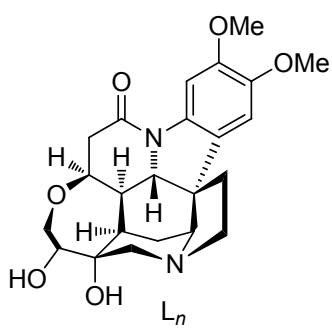




\section{4 金属-羧酸/磺酸共催化}

羧酸因能提供质子而多被作为 Brønsted 酸催化有 机反应. 2014 年, 杨罗研究组 ${ }^{[54]}$ 报道了 $\mathrm{Cu}$ 和特戊酸共 催化的交叉脱氢偶联反应, 如 Scheme 16 所示, 在特戊 酸的催化作用下反应物 2-甲基氮杂芳烃 109 转化为亲核 性的烯胺, 反应物四氢异喹啉被氧气氧化为亲电性的亚 胺正离子并被 $\mathrm{Cu}$ 催化剂配位稳定, 随即与烯胺中间体 通过类 Mannich 反应, 实现了两个 $\mathrm{sp}^{3}$ 杂化的 $\mathrm{C}-\mathrm{H}$ 键 之间的交叉脱氢偶联.

对甲苯磺酸作为一种强酸类催化剂被广泛用于有 机合成中. 近期, Alcaide 研究组 ${ }^{[55]}$ 以 $\mathrm{Au}$ 和对甲苯磺酸 的共催化体系, 炔丙基过氧化氢与氨基吡唑为原料, 在 微波辐射下合成了稠氮杂环化合物, 如 Scheme 17 所示. 该反应的机理可能为: 炔丙基过氧化氢在 $\mathrm{AuClPPh}_{3}$ 的
催化作用下通过重排异构化为 $\alpha, \beta$-不饱和酮 116, 中间 体 116 与氨基吡唑通过氮杂的迈克尔加成反应生成 $\beta$ 氨基酮 117, 117 在对甲苯磺酸的质子化作用下与氨基发 生分子内的缩合反应生成稠氮杂环化合物 119.

因金属催化剂和有机催化剂自身类型非常丰富，以 致二者组合的金属-有机共催化体系较为多样，如上文 所述的金属-有机胺共催化 ${ }^{[56 ~ 59]}$ 、金属-磷酸共催化 ${ }^{[60]}$ 、 金属-硫艮共催化、金属-羧酸/磺酸共催化 ${ }^{[61]}$ 等体系. 金 属-有机共催化体系具有金属催化剂和有机催化剂的双 重优点，在有机合成中将会有更广泛的应用.

\section{4 结论与展望}

共催化作为一类新颖的催化模式提供了一种更简 洁、更高效的催化途径，使得许多新的化学转化得以实<smiles>[H][R11]1ccc2c(c1)[X]=CC=C(C)N=2</smiles>

109

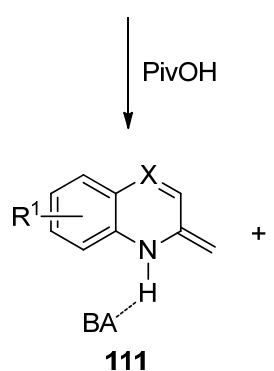

111<smiles>[R]c1ccc(N2CCc3ccccc3C2)cc1</smiles>

110

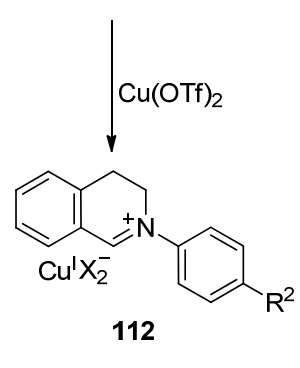

$\mathrm{Cu}(\mathrm{OTf})_{2}(5 \mathrm{~mol} \%)$

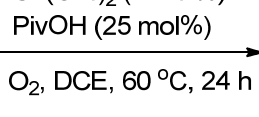<smiles>[R9]c1ccc(N2CCc3ccccc3C2Cc2ccc3cc[R1]cc3c2)cc1</smiles>
交叉脱氢偶联

$\mathrm{R}^{1}=\mathrm{H}, 6-\mathrm{OCH}_{3}, 6-\mathrm{Cl}, 6-\mathrm{I}, 6-\mathrm{CF}_{3}$, $6-\mathrm{CH}_{3}, 6-\mathrm{F}, 6-\mathrm{Br}, 6-\mathrm{Ph}, 6-\mathrm{NO}_{2}$

$\mathrm{X}=\mathrm{CH}, \mathrm{N}$

$\mathrm{R}^{2}=\mathrm{CH}_{3}, \mathrm{OCH}_{3}, \mathrm{~F}, \mathrm{Cl}, \mathrm{Br}$

图式 16 铜和特戊酸共催化的交叉脱氢偶联反应

Scheme 16 Cross dehydrogenation coupling reaction catalyzed by $\mathrm{Cu}$ and pivalic acid
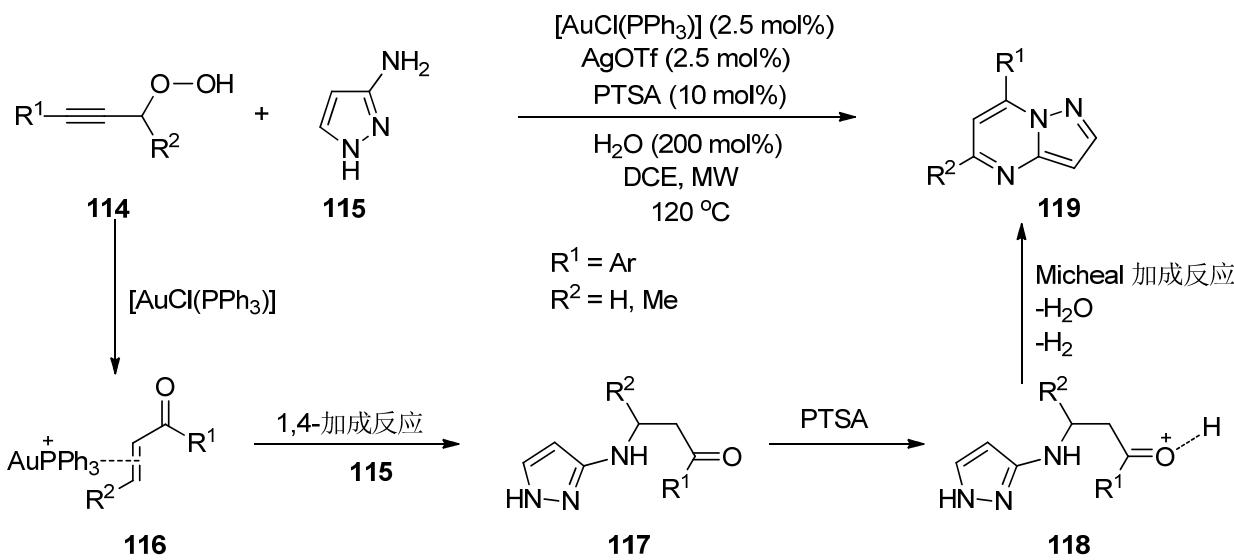

图式 17 微波辐射下金和对甲苯磺酸共催化合成稠氮杂环化合物

Scheme 17 Microwave-assisted synthesis of fused azaheterocycles catalyzed by Au and $p$-toluenesulfonic acid 
现. 随着共催化概念的不断深入, 其优越性也不断得到 体现，本文从金属-金属共催化、有机一有机共催化和金 属一有机共催化三种类型, 综述了近年来共催化领域中 新颖的、重要的研究成果, 特别是金属和有机小分子的 共催化体系, 由于结合了金属催化剂和有机催化剂两者 的优点已成为有机合成领域的研究热点. 共催化体系具 有简洁、高效和高选择性等优点, 但因中间态较为复杂, 对其结构和机理还需进行更深入的研究, 以便将来开发 和拓展更多高效、新颖、多样的催化体系.

\section{References}

[1] Allen, A. E.; MacMillan, D. W. C. Chem. Sci. 2012, 3, 633.

[2] Zhou. J. Chem. Asian J. 2010, 5, 422.

[3] Su, Y. J.; Jia, W.; Jiao, N. Synthesis 2011, 1678.

[4] Dijk, E. W.; Panella, L.; Pinho, P.; Naasz, R.; Meetsma, A.; Minnaard, A. J.; Feringa, B. L. Tetrahedron 2004, 60, 9687.

[5] Wang, X. H.; Yao, Z. L.; Dong, S. L.; Wei, F.; Wang, H.; Xu, Z. H. Org. Lett. 2013, 15, 2234.

[6] Zeng, J.; Tan, Y. J.; Leow, M. L.; Liu, X. W. Org. Lett. 2012, 14, 4386.

[7] Wang, X. B.; Wang, Z. Y. Tetrahedron 2014, 70, 6728.

[8] Nakao, Y.; Kanyiva, K. S.; Hiyama, T. J. Am. Chem. Soc. 2008, $130,2448$.

[9] Shih, W. C.; Chen, W. C.; Lai, Y. C.; Yu, M. S.; Ho, J. J.; Yap, G. P. A.; Ong, T. G. Org. Lett. 2012, 14, 2046.

[10] Fan, B. M.; Li, S. F.; Chen, H. L.; Lu, Z. W.; Liu, S. S.; Yang, Q. J.; Yu, L.; Xu, J. B.; Zhou, Y. Y.; Wang, J. Adv. Synth. Catal. 2013, $355,2827$.

[11] Guo, Z. Q.; Cai, M.; Jiang, J.; Yang, L. P.; Hu, W. H. Org. Lett. 2010, 12,652

[12] Wang, H. G.; Wang, Y.; Peng, C. L.; Zhang, J. C.; Zhu, Q. J. Am. Chem. Soc. 2010, 132, 13217.

[13] Shirakawa, E.; Ikeda, D.; Masui, S.; Yoshida, M.; Hayashi, T. J. Am. Chem. Soc. 2012, 134, 272.

[14] Onodera, G.; Nishibayashi, Y.; Uemura, S. Angew. Chem., Int. Ed. 2006, 45, 3819.

[15] Qin, C.; Feng, P.; Ou, Y.; Shen, T.; Wang, T.; Jiao, N. Angew. Chem., Int. Ed. 2013, 52, 7850.

[16] Zeng, J.; Liu, K. M.; Duan, X. F. Org. Lett. 2013, 15, 5342.

[17] Trost, B. M.; Machacck, M. R.; Faulk, B. D. J. Am. Chem. Soc. 2006, $128,6745$.

[18] Dalko, P. I.; Moisan, L. Angew. Chem., Int. Ed. 2004, 43, 5138.

[19] List, B.; Lerner, R. A.; Barbas III, C. F. J. Am. Chem. Soc. 2000, 122, 2395.

[20] Bastida, D.; Liu, Y. K.; Tian, X.; Adan, E. E.; Melchiorre, P. Org. Lett. 2013, 15, 220.

[21] De, C. K.; Seidel, D. J. Am. Chem. Soc. 2011, 133, 14538.

[22] Yang, X. Y.; Phipps, R. J.; Toste, F. D. J. Am. Chem. Soc. 2014, $136,5225$.

[23] Enders, D.; Niemeier, O.; Balensiefer, T. Angew. Chem., Int. Ed. 2006, $45,1463$.

[24] Takikawa, H.; Hachisu, H.; Bode, J. W.; Suzuki, K. Angew. Chem., Int. Ed. 2006, 45, 3492.

[25] Liu, Q.; Rovis, T. J. Am. Chem. Soc. 2006, 128, 2552.

[26] Li, G. Q.; Dai, L. X.; You, S. L. Chem. Commun. 2007, 852.

[27] Qu, M. N.; He, J. M. Chin. J. Org. Chem. 2011, 31, 1388 (in Chi- nese).

(屈孟男，何金梅，有机化学, 2011, 31, 1388.)

[28] Lathrop, S. P.; Rovis, T. J. Am. Chem. Soc. 2009, 131, 13628.

[29] Chiang, P. C.; Kaeobamrung, J.; Bode, J. W. J. Am. Chem. Soc. 2007, 129, 3520 .

[30] He, M.; Struble, J. R.; Bode, J. W. J. Am. Chem. Soc. 2006, 128, 8418.

[31] Zhao, X. D.; DiRocco, D. A.; Rovis, T. J. Am. Chem. Soc. 2011, $133,12466$.

[32] Lu, L. Q.; Cao, Y. J.; Liu, X. P.; An, J.; Yao, C. J.; Ming, Z. H.; Xiao, W. J. J. Am. Chem. Soc. 2008, 130, 6946.

[33] Youn, S. W.; Song, H. S.; Park, J. H. Org. Lett. 2014, 16, 1028.

[34] Park, Y. J.; Park, J. W.; Jun, C. H. Acc. Chem. Res. 2008, 41, 222.

[35] Shao, Z. H.; Zhang, H. B. Chem. Soc. Rev. 2009, 38, 2745.

[36] Zhong, C.; Shi, X. D. Eur. J. Org. Chem. 2010, 2999.

[37] Ibrahem, I.; Cordova, A. Angew. Chem., Int. Ed. 2006, 45, 1952.

[38] Zhao, X.; Liu, D.; Xie, F.; Liu, Y.; Zhang, W. Org. Biomol. Chem. 2011, 9, 1871.

[39] Ibrahem, I.; Ma, G. N.; Afewerki, S.; Cordova, A. Angew. Chem., Int. Ed. 2013, 52, 878 .

[40] Ma, G. N.; Afewerki, S.; Deiana, L.; Nieto, C. P.; Liu, L. F.; Sun, J. L.; Ibrahem, I.; Cordova, A. Angew. Chem., Int. Ed. 2013, 52, 6050.

[41] Guo, C. S.; Du, Y. H.; Huang, Z. Z. Chem. Commun. 2011, 47, 3995.

[42] Beletskiy, E. V.; Sudheer, C.; Douglas, C. J. J. Org. Chem. 2012, $77,5884$.

[43] Shibata, Y.; Tanaka, K. J. Am. Chem. Soc. 2009, 131, 12552.

[44] Tanaka, K.; Shibata, Y.; Suda, T.; Hagiwara, Y.; Hirano, M. Org. Lett. 2007, 9, 1215.

[45] Shibahara, F.; Bower, J. F.; Krische, M. J. J. Am. Chem. Soc. 2008, 130,14120 .

[46] Hong, Y. T.; Barchuk, A.; Krische, M. J. Angew. Chem., Int. Ed. 2006, 45, 6885 .

[47] Krautwald, S.; Sarlah, D.; Schafroth, M.; Carreira, E. Science 2013, 340, 1065.

[48] Qiu, H.; Li, M.; Jiang, L. Q.; Lv, F. P.; Zan, L.; Zhai, C. W.; Doyle, M.; Hu, W. H. Nat. Chem. 2012, 4, 733.

[49] Qiu, L.; Guo, X.; Ma, C. Q.; Qiu, H.; Liu, S. Y.; Yang, L. P.; Hu, W. H. Chem. Commun. 2014, 50, 2196.

[50] Jiang, J.; Xu, H. D.; Xi, J. B.; Ren, B. Y.; Lv, F. P.; Guo, X.; Jiang, L. Q.; Zhang, Z. Y.; Hu, W. H. J. Am. Chem. Soc. 2011, 133, 8428.

[51] Jiang, J.; Ma, X. C.; Ji, C. G.; Guo, Z. Q.; Shi, T. D.; Liu, S. Y.; Hu, W. H. Chem. Eur. J. 2014, 20, 1505.

[52] Cai, Q.; Zhao, Z. A.; You, S. L. Angew. Chem., Int. Ed. 2009, 48, 7428.

[53] Kim, H. Y.; Oh, K. Org. Lett. 2011, 13, 1306.

[54] Wang, F. F.; Luo, C. P.; Deng, G. J.; Yang, L. Green Chem. 2014, $16,2428$.

[55] Alcaide, B.; Almendros, P.; Quiros, M. T. Chem. Eur. J. 2014, 20, 3384.

[56] Xie, J.; Huang, Z. Z. Angew. Chem., Int. Ed. 2010, 49, 10181.

[57] Patil, N. T.; Raut, V. S. J. Org. Chem. 2010, 75, 6961.

[58] Arai, T.; Yamamoto, Y. Org. Lett. 2014, 16, 1700.

[59] Xiao, J. Org. Lett. 2012, 14, 1716.

[60] Yu, S. Y.; Zhang, H.; Gao, Y.; Mo, L.; Wang, S. Z.; Yao, Z. J. J. Am. Chem. Soc. 2013, 135, 11402 .

[61] Ni, Y.; Guo, X.; Hu, W. H.; Liu, S. Y. Chin. J. Org. Chem. 2014, 34, 107 (in Chinese)

(倪懿，郭金金，胡文浩，刘顺英，有机化学, 2014, 34, 107.) 